\title{
Обнаружение и количественное определение адсорбированного хлора на поверхностях конструкционных материалов
}

\author{
(С2020 Кузнецова Е.С., Пыцкий И.С., Буряк А.К. \\ Федеральное Государственное Бюджетное Учреждение Науки Институт физической химии \\ и электрохимии имени А.Н. Фрумкина РАН, Москва
}

Поступила в редакцию 17.09.2020 г.

DOI: $10.17308 /$ sorpchrom.2020.20/3051

В работе приведены результаты исследования возможностей количественного анализа с использованием метода МАЛДИ масс-спектрометрии. Рассмотрена важная практическая задача обнаружения и определения хлора на поверхностях алюминиевых конструкционных материалов после атмосферной коррозии. Обнаружено, что по хлорид иону обнаружение хлора возможно только до $10^{-}$ 12 моль/мм², что мало соответствует практическим требованиям. Показано, что аналитические характеристики при определении хлора могут быть значительно улучшены при проведении дериватизации поверхности нитратом серебра. Образующиеся кластерные ионы снижают предел чувствительности до $10^{-18}$ моль/мм² и расширяют динамический диапазон до трёх порядков по концентрации. Также показано, что используемый в работе метод может быть распространён и на обнаружение других галогенов на различных поверхностях, может применяться в различных областях науки и промышленности. Например, для поиска хлора на поверхности различных конструкционных материалов, определения локальных концентраций хлора в конкретной точке, построения диаграмм распределения хлора по поверхностям различных конструкционных материалов, изучения кинетики сорбции хлора при экспериментах по атмосферной коррозии, а также для поиска нарушений морфологии поверхности по распределению адсорбированного хлора на поверхности.

Ключевые слова: масс-спектрометрия, хлор, хлориды, кластерообразование, конструкционные материалы, количественный анализ, поверхность.

\section{Введение}

Методы поверхностной масс-спектрометрии являются одними из самых современных методов исследования различных материалов и изучения свойств поверхности [1-3]. Одной из часто используемых разновидностей метода является матрично-активированная лазерная десорбция/ионизация (МАЛДИ), которая включает в себя все достоинства масс-спектрометрических методов (высокую чувствительность, универсальность, возможность совмещения с хроматографическими методами, хотя и в офф-лайн - режиме). В тоже время МАЛДИ имеет принципиальные отличия от другой масс-спектрометрии, так как является методом исследования поверхностей и имеет дело не с объёмом растворов, а с двумерными образцами поверхности. Большая часть исследований МАЛДИ связана с исследованием образцов, которые наносятся на специально подготовленную поверхность из растворов и высушиваются на воздухе. Таким образом исследуют различные высокомолекулярные соединения, которые ионизируются без разрушения благодаря «мягкой» лазерной ионизации [4-6]. 
Ещё одним популярным аспектом исследований методом МАЛДИ является применение его для исследования распределения соединений по поверхности тонких тканей или плёнок. Обычно в качестве таких образцов применяют срезы живых тканей различных растений или животных [7-9]. Такие работы пользуются завидной популярностью благодаря применению современных технологий обработки большого объёма данных и позволяют проводить исследование распределения таких соединений по различным поверхностям и внутри живых тканей. Авторами было предложено использование этого способа для исследования различных металлических поверхностей [10-11]. При исследовании образцов различных металлов (меди, цинка, железа, алюминия и других) после атмосферной коррозии часто на поверхности обнаруживают следовые количества хлора адсорбированного из атмосферы. При этом его абсолютные количества на поверхности установить без какой-либо калибровки не представляется возможным. Целью данной работы являлось установление характера зависимости между сигналами хлора и его количества на поверхности. Поиск пределов детектирования и динамического диапазона определения хлора методом МАЛДИ на металлических поверхностях. Для сравнения будут также использоваться способы обнаружения других галогенов на поверхности. Будут установлены относительные чувствительности по различным галогенам на поверхности различных материалов.

\section{Экспериментальная часть}

Для калибровки прибора использовали хлорид, бромид и иодид калия (Реахим, Россия). Для приготовления растворов использовали воду деионизированную $\Omega=18$ Мом. Также использовали раствор нитрата серебра (Реахим, Россия) для улучшения аналитических характеристик метода. В табл. 1 указаны типы поверхностей и галогенсодержащие соединения, которые использовали для изучения аналитических характеристик метода.

Таблица 1. Соединения, используемые для исследования на различных металлических материалах («да» и «нет» поясняет проводилось ли исследование данных соединений на данной поверхности).

Table 1. Compounds used for testing on various metallic materials ("yes" and "no" denotes whether these compounds were tested on a given surface).

\begin{tabular}{|c|c|c|c|}
\hline & $\begin{array}{c}\text { Инертная поверхность } \\
\text { нержавеющей стали }\end{array}$ & $\begin{array}{c}\text { Сплав алюминиевый } \\
\text { Ад-0 }\end{array}$ & $\begin{array}{c}\text { Сплав алюми- } \\
\text { ниевый АМг-6 }\end{array}$ \\
\hline $\begin{array}{c}\text { Галогениды калия } \\
\left(10^{-2}-10^{-5} \mathrm{M}\right)\end{array}$ & Да & Да & Да \\
\hline $\begin{array}{c}\text { Галогениды калия } \\
\left(10^{-7}-10^{-12} \mathrm{M}\right)\end{array}$ & Да & Да & Да \\
\hline $\begin{array}{c}\text { Нитрат серебра } \\
\left(10^{-2}-10^{-5} \mathrm{M}\right)\end{array}$ & Нет & Да & Да \\
\hline $\begin{array}{c}\text { Нитрат серебра } \\
\left(10^{-7}-10^{-12} \mathrm{M}\right)\end{array}$ & Да & Да & Да \\
\hline $\begin{array}{c}\text { Галогениды серебра } \\
\left(10^{-2}-10^{-5} \mathrm{M}\right)\end{array}$ & Да & Да & Да \\
\hline $\begin{array}{c}\text { Галогениды серебра } \\
\left(10^{-7}-10^{-12} \mathrm{M}\right)\end{array}$ & Да & Да & \\
\hline
\end{tabular}

В качестве образца сравнения использовали инертную специально обработанную плашку из нержавеющей стали (Steel plate 384, Bruker, Германия). Также ис- 
следовали чистые образцы алюминиевых сплавов Амг-6 И Ад-0 в качестве модельных соединений содержащих атмосферный хлор. Исследование проводили на массспектрометре МАЛДИ Bruker Daltonic Ultraflex II (Bruker, Германия), оснащённым азотным лазером с рабочей длиной волны $\lambda=337$ нм. Частота импульсов варьировалась от 20 до 200 Гц, энергия одного импульса варьировалась от 10 до 110 мкДж. Подбор конкретных условий для каждого опыта подбирался индивидуально в процессе эксперимента.

\section{Обсуждение результатов}

В таблице 2 приведены средние интенсивности пиков $\mathrm{Cl}^{-}$в масс-спектрах различных хлорсодержащих солей.

Таблица 2. Интенсивности пиков солей (m/z пика) в масс-спектрах, зарегистрированных с различных поверхностей.

Table 2. Intensities of salt peaks (m/z peak) in mass spectra recorded from various surfaces.

\begin{tabular}{|c|c|c|c|}
\hline $\begin{array}{l}\text { Интенсивность, } \\
\text { усл. ед. } \\
\end{array}$ & $\begin{array}{c}\text { Инертная поверхность } \\
\text { нержавеющей стали }\end{array}$ & $\begin{array}{c}\text { Сплав алюминиевый } \\
\text { Ад-0 }\end{array}$ & $\begin{array}{c}\text { Сплав алюминие- } \\
\text { вый АМг-6 } \\
\end{array}$ \\
\hline $\begin{array}{c}\text { Галогениды калия } \\
\left(10^{-2}-10^{-5} \mathrm{M}\right)\end{array}$ & $\left(\mathrm{Cl}^{-} ; 35\right.$ Да) 500-600 & $\left(\mathrm{Cl}^{-} ; 35\right.$ Да) 150-200 & $\left(\mathrm{Cl}^{-} ; 35\right.$ Да) 100-150 \\
\hline $\begin{array}{c}\text { Галогениды калия } \\
\left(10^{-7}-10^{-12} \mathrm{M}\right)\end{array}$ & $\left(\mathrm{Cl}^{-} ; 35\right.$ Да) 50-100 & $\left(\mathrm{Cl}^{-} ; 35\right.$ Да) 30-50 & $\left(\mathrm{Cl}^{-} ; 35\right.$ Да) 30-50 \\
\hline $\begin{array}{l}\text { Нитрат серебра } \\
\left(10^{-2}-10^{-5} \mathrm{M}\right)\end{array}$ & - & - & - \\
\hline $\begin{array}{l}\text { Нитрат серебра } \\
\left(10^{-7}-10^{-12} \mathrm{M}\right)\end{array}$ & - & - & - \\
\hline 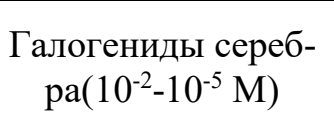 & $\begin{array}{c}\left(\mathrm{Cl}^{-} ; 35 \text { Да) } 60-110\right. \\
\left(\mathrm{Ag}_{2} \mathrm{Cl}^{-} ; 249 \text { Да }\right) \\
>10000\end{array}$ & $\begin{array}{c}\left(\mathrm{Cl}^{-} ; 35 \text { Да) 20-60 }\right. \\
\left(\mathrm{Ag}_{2} \mathrm{Cl}^{-} ; 249 \text { Да) }>10000\right.\end{array}$ & $\begin{array}{c}\left(\mathrm{Cl}^{-} ; 35 \text { Да }\right) 20-40 \\
\left(\mathrm{Ag}_{2} \mathrm{Cl}^{-} ; 249 \text { Да }\right) \\
>10000\end{array}$ \\
\hline $\begin{array}{c}\text { Галогениды сереб- } \\
\text { ра }\left(10^{-7}-10^{-12} \mathrm{M}\right)\end{array}$ & $\begin{array}{c}\left(\mathrm{Cl}^{-} ; 35 \text { Да) } 20-30\right. \\
\left(\mathrm{Ag}_{2} \mathrm{Cl}^{-} ; 249 \text { Да) }\right. \\
5000-6000\end{array}$ & $\begin{array}{c}\left(\mathrm{Cl}^{-} ; 35 \text { Да) } 20-30\right. \\
\left(\mathrm{Ag}_{2} \mathrm{Cl}^{-} ; 249 \text { Да }\right. \\
) 3500-4000\end{array}$ & $\begin{array}{c}\left(\mathrm{Cl}^{-} ; 35 \text { Да) } 20-30\right. \\
\left(\mathrm{Ag}_{2} \mathrm{Cl}^{-} ; 249 \text { Да) }\right. \\
3500-4000\end{array}$ \\
\hline
\end{tabular}

Из таблицы видно, что чувствительность по хлору метода МАЛДИ крайне низка и зависимость высоты пика от концентрации плохо прослеживается. Следует отметить, что несмотря на это установленный предел чувствительности по хлору составляет $10^{-5} \mathrm{M}$ раствора, что при пересчёте на поверхность нержавеющей стали составляет около $5 \cdot 10^{-12}$ моль/мм². При этом на поверхностях конструкционных материалов интенсивность пиков значительно уменьшается и чувствительность по хлору составляет не более $10^{-12}$ моль/мм². Следует отметить, что ситуация значительно изменяется при нанесении на исследуемую поверхность нитрата серебра. Авторами было показано, что при нанесении на любую поверхность, содержащую ионы хлора образуются нерастворимые соли серебра, образующие высокоинтенсивные пики хлорсодержащих кластерных ионов [10], которые также могут быть использованы для количественного определения хлора на поверхности.

Благодаря высокой интенсивности пиков хлорида серебра предел чувствительности может быть снижен на 6 порядков и достигать $10^{-18}$ моль/мм². 


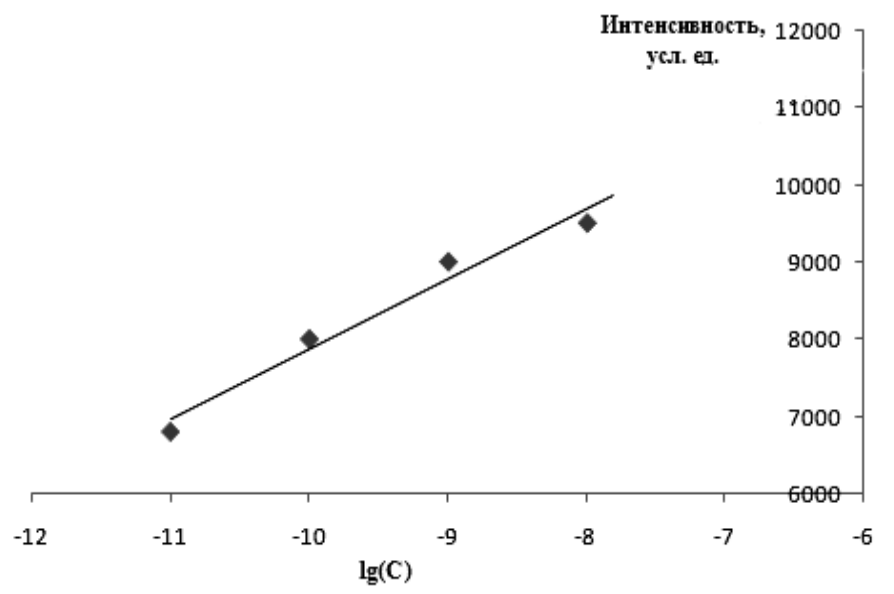

Рис. 1. Зависимость интенсивности пика кластерного иона хлорида серебра $\left(\mathrm{m} / \mathrm{z}=320.7\right.$ Да) от логарифма концентрации (в моль/дм $\left.{ }^{3}\right)$ хлора при использовании режима регистрации отрицательных ионов.

Fig. 1. Dependence of the intensity of the peak of the silver chloride cluster ion $(\mathrm{m} / \mathrm{z}=320.7 \mathrm{Da})$ on the logarithm of the concentration $(\mathrm{mol} / \mathrm{l})$ of chlorine using the mode of registration of negative ions.

При исследовании возможности количественного определения хлора был построен график зависимости интенсивности сигнала кластерного иона $\mathrm{Ag}_{2} \mathrm{Cl}_{3}{ }^{-}$от количества хлора на поверхности. Уравнение графика у $=(910 \pm 30) x+(16970 \pm 120)$ при доверительной вероятности $\mathrm{P}=0.95$ и $\mathrm{n}=6$. Показано что график спрямляется при логарифмировании оси абсцисс. Линейность концентрации составляет 3 и более порядка и позволяет проводить полуколичественный анализ хлора в любой точке исследуемой поверхности в динамическом диапазоне от $10^{-18}$ до $10^{-15}$ моль $/ \mathrm{Mm}^{2}$.

При исследовании других галогенидов серебра были построены графики изображённые на рисунке 2 и 3 . Для бромидов уравнение имеет вид у $=(2600 \pm 100) x$ $+(32950 \pm 180)$ при доверительной вероятности $\mathrm{P}=0.95$ и $\mathrm{n}=6$.

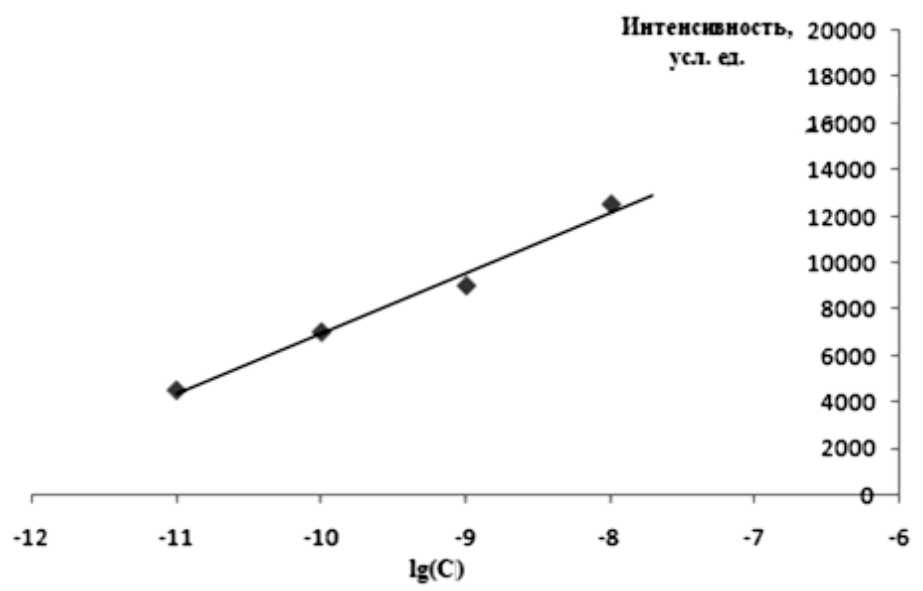

Рис. 2. Зависимость интенсивности пика кластерного иона бромида серебра $\mathrm{c} n=2\left(\mathrm{~m} / \mathrm{z}=454.6\right.$ Да) от логарифма концентрации (в моль/дм $\left.{ }^{3}\right)$ брома при использовании режима регистрации отрицательных ионов.

Fig. 2. Dependence of the intensity of the peak of the silver bromide cluster ion with $n=2(m / z=454.6 \mathrm{Da})$ on the logarithm of the concentration $(\mathrm{mol} / \mathrm{l})$ of bromine using the mode of registration of negative ions. 


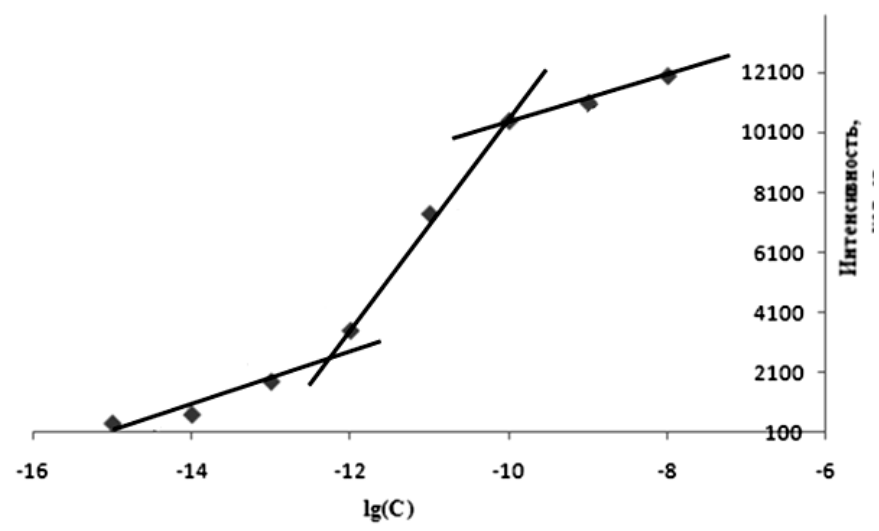

Рис. 3. Зависимость интенсивности пика кластерного иона иодида серебра $\mathrm{c} \mathrm{n}=2\left(\mathrm{~m} / \mathrm{z}=596.5\right.$ Да) от логарифма концентрации (в моль/дм $\left.{ }^{3}\right)$ иода при использовании режима регистрации отрицательных ионов.

Fig. 3. Dependence of the intensity of the peak of the silver iodide cluster ion with $\mathrm{n}=2(\mathrm{~m} / \mathrm{z}=596.5 \mathrm{Da})$ on the logarithm of the concentration $(\mathrm{mol} / \mathrm{l})$ of iodine using the mode of registration of negative ions.

Было показано, что кластеры бромидов и иодидов серебра более чувствительны к изменению концентрации соответствующих галогенов при этом, однако, не удаётся добиться линейности калибровочного графика для определения концентраций иода на поверхностях. В то же время, пределы обнаружения для других галогенов не хуже, чем для хлора и позволяют использовать метод для обнаружения следовых количеств галогенов на различных поверхностях.

\section{Заключение}

Авторами разработан метод определения следовых количеств адсорбированного на поверхности хлора методом МАЛДИ. Показано, что при значительных ко-

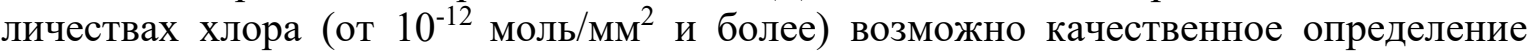
хлора по наличию пика $\mathrm{Cl}^{-}$в масс-спектрах, зарегистрированных в различных точках изучаемой поверхности. Однако для работы в более низких и более вероятных диапазонах концентраций необходимо применять модифицирование поверхности нитратом серебра. В этом случае при наличии на поверхности следов хлора в массспектре регистрируются высокоинтенсивные пики кластерных ионов галогенидов серебра, которые являются признаком присутствия хлора на поверхности. Так возможна калибровка прибора по хлору таким способом. Из работы видно, что пределы обнаружения могут достигать $10^{-18}$ моль/мм². Метод прост в применении и может быть использован для: поиска хлора на поверхности различных конструкционных материалов; определения локальных концентраций хлора в конкретной точке; построения диаграмм распределения хлора по поверхностям различных конструкционных материалов; изучения кинетики сорбции хлора при экспериментах по атмосферной коррозии; поиска нарушений морфологии поверхности по распределению адсорбированного хлора на поверхности.

Кроме этого, показано, что метод может быть развит и для поиска следовых количеств брома и иода, однако, в связи с неочевидным практическим применением эти исследования будут продолжены в будущих работах.

Авторы выражают благодарность Министерству образования и науки Российской Федерации за финансовую поддержку. 


\title{
Список литературы/References
}

1. Ryan D.J., Spraggins J.M., Caprioli R.M., Current opinion in chemical biology, 2019, Vol. 48, pp. 64-72.

2. Feucherolles M., Parasites \& Vectors, 2019, Vol. 12, No 1, pp. 1-13.

3. Shimizu A., Hase R., Suzuki D., Toguchi A. et al., Journal of infection and chemotherapy, 2019, Vol. 25, No 2, pp. 141-146.

4. Ahmad R., Jang H., Batule B.S., Park H.G., Analytical chemistry, 2017, Vol. 89, No 17, pp. 8966-8973.

5. Liu Z., Zhang P., Kästner L., Volmer D.A., Journal of Mass Spectrometry, 2019, Vol. 54, No 11, pp. 878-884.

6. Guran R., Vanickova L., Horak V., Krizkova S. et al., PloS one, 2017, Vol. 12, No 12, pp. 189-205.
7. Ryan D.J., Spraggins J.M., Caprioli R.M., Current opinion in chemical biology, 2019, Vol. 48, pp. 64-72.

8. Schwamborn K., Kriegsmann M., Weichert W., Biochimica et Biophysica Acta (BBA)Proteins and Proteomics, 2017, Vol. 1865, No 7, pp. 776-783.

9. Touve M.A., Carlini A.S., Gianneschi N.C., Nature communications, 2019, Vol. 10, No 1, pp. 1-12.

10. Pytskii I.S., Petukhova G.A., Kuznetsova E.S., Buryak A.K. Surface innovations, 2017, Vol. 5, No 3, pp. 179-187.

11. Kuznetsova E.S., Pytskii I.S., Buryak A.K., Sorbtsionnye $i$ khromatograficheskie protsessy, 2018, Vol. 18, No 2, pp. 238-242.

\section{Detection and quantitative determination of chlorine on the surfaces of construction materials}

\author{
(C) 2020 Kuznetsova E.S., Pytsky I.S., Buryak A.K. \\ A. N. Frumkin Institute of Physical Chemistry and Electrochemistry of the Russian Academy of Sciences, \\ Moscow, Russian Federation
}

The study presents the results of an investigation into the possibilities of quantitative analysis using the MALDI method. An important practical problem was considered in relation to the detection and determination of chlorine on the surfaces of aluminium structural materials after atmospheric corrosion. It was found that chlorine detection by chloride ions is possible only up to $10^{-12} \mathrm{~mol} / \mathrm{mm}^{2}$, which hardly meets practical requirements. It was shown that the analytical characteristics in the determination of chlorine can be significantly improved by derivatizing the surface with silver nitrate. The resulting cluster ions reduce the sensitivity limit to $10^{-18} \mathrm{~mol} / \mathrm{mm}^{2}$ and expand the dynamic range to three orders of magnitude in concentration. It was also shown that the method can be extended to detect other halogens on various surfaces. The method can be applied in various fields of science and industry. For example, the method can be used in the search for chlorine on the surface of various structural materials, for the determination of local chlorine concentrations at a specific point, for plotting chlorine distribution diagrams over the surfaces of various structural materials, for studying the kinetics of chlorine sorption in experiments on atmospheric corrosion, and also for the search for impairments of surface morphology based on the distribution of adsorbed chlorine on the surface.

Keywords: mass spectrometry, chlorine, chlorides, clustering, construction materials, quantitative analysis, surface.

Кузнецова Елена Сергеевна - старший научный сотрудник лаборатории синтеза и исследования сорбентов, к.х.н. Институт физической химии и электрохимии имени А.Н. Фрумкина РАН, Москва

Пыцкий Иван Сергеевич - старший научный сотрудник лаборатории физико-химических основ хроматографии и хромато-масс-спектрометрии, к.х.н. Институт физической химии и электрохимии имени А.Н. Фрумкина РАН, Москва

Буряк Алексей Константинович - заведующий лабораторией физико-химических основ хроматографии и хромато-масс-спектрометрии, проф, д.Х.н. Институт физической химии и электрохимии имени А.Н. Фрумкина РАН, Москва
Kuznetsova Elena S. - PhD (chemistry), laboratory of synthesis and investigation of sorbents, Institute of Physical chemistry and electrochemistry, Moscow

Pytskii Ivan S. - PhD (chemistry), laboratory of physicalchemical basics of chromatography and chromato-massspectrometry, Institute of Physical chemistry and electrochemistry, Moscow. E-mail: ivanpic4586@gmail.com

Buryak Alexey K. - prof., grand $\mathrm{PhD}$ (chemistry), laboratory of physical-chemical basics of chromatography and chromato-mass-spectrometry Institute of Physical chemistry and electrochemistry, Moscow 